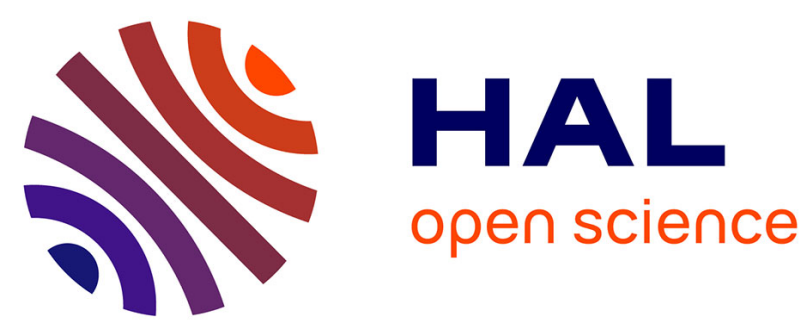

\title{
Unraveling the hydration-induced ground-state change of AtO+ by relativistic and multiconfigurational wave-function-based methods
}

Dumitru-Claudiu Sergentu, Florent Réal, Gilles F Montavon, Nicolas Galland, Rémi Maurice

\section{To cite this version:}

Dumitru-Claudiu Sergentu, Florent Réal, Gilles F Montavon, Nicolas Galland, Rémi Maurice. Unraveling the hydration-induced ground-state change of $\mathrm{AtO}+$ by relativistic and multiconfigurational wave-function-based methods. Physical Chemistry Chemical Physics, 2016, 18, pp.32703-32712. 10.1039/C6CP05028J . in2p3-01447927

\section{HAL Id: in2p3-01447927 \\ https://hal.in2p3.fr/in2p3-01447927}

Submitted on 2 Dec 2021

HAL is a multi-disciplinary open access archive for the deposit and dissemination of scientific research documents, whether they are published or not. The documents may come from teaching and research institutions in France or abroad, or from public or private research centers.
L'archive ouverte pluridisciplinaire HAL, est destinée au dépôt et à la diffusion de documents scientifiques de niveau recherche, publiés ou non, émanant des établissements d'enseignement et de recherche français ou étrangers, des laboratoires publics ou privés. 


\title{
Journal Name
}

\section{ARTICLE TYPE}

\section{Cite this: DOI: $10.1039 / x x x x x x x x x x$ \\ Unraveling the hydration-induced ground-state change of $\mathrm{AtO}^{+}$with relativistic and multiconfigu- rational wave-function-based methods ${ }^{\dagger}$}

\author{
Dumitru-Claudiu Sergentu, ${ }^{a, b}$ Florent Réal, ${ }^{c}$ Gilles Montavon, ${ }^{a}$ Nicolas Galland, ${ }^{* b}$ and \\ Rémi Maurice*a
}

Received Date

Accepted Date

DOI: 10.1039/xxxxxxxxxx

www.rsc.org/journalname
$\mathrm{The} \mathrm{AtO}^{+}$cation is one of the main chemical forms that appear in the astatine Pourbaix diagram. This form can react with closed-shell species in solution while in the gas phase, it has a spintriplet ground spin-orbit-free (SOF) state. Spin-orbit coupling (SOC) mixes its $M_{S}=0$ component with the ${ }^{1} \Sigma^{+}$singlet-spin component while keeping an essentially-spin-triplet SOC ground-state. Therefore, it was suggested that $\mathrm{AtO}^{+}$undergoes a hydration-induced ground-state change to explain its reactivity in solution with closed-shell species [J. Phys. Chem. B, 2013, 117, 52065211]. In this work, we track the natures of the low-lying SOF and SOC states when the hydration sphere of $\mathrm{AtO}^{+}$is stepwise increased, with relativistic and multiconfigurational wave-functionbased methods. This work clarifies previous studies by (i) giving additional arguments justifying a solvation-induced ground-state change in this system and (ii) clearly identifying for the first time the nature of the involved SOF and SOC many-electron states. Indeed, we find at the SOF level that $\mathrm{AtO}^{+}$undergoes a ground-state reversal between ${ }^{3} \Sigma^{-}$and the closed-shell component of ${ }^{1} \Delta$, which leads to an essentially-spin-singlet and closed-shell SOC ground-state. This explains the observed reactivity of $\mathrm{AtO}^{+}$with closed-shell species in solution.

\section{Introduction}

In most cases, environment effects only moderately affect the nature of the ground-state of molecular systems. This is why it is usually wise to study such systems in vacuum to get a first picture on their ground-state electronic structures. However, in some peculiar cases, such a picture may be qualitatively incorrect, e.g. when an environment-induced ground-state reversal occurs. Molecular systems which are prone to such phenomena typically exhibit small energy gaps between their ground-state and one or more excited states in the gas phase, as in actinide-containing systems. For instance, $\mathrm{CUO}$ and $\mathrm{UO}_{2}$ are notorious examples that exhibit ground-state reversals upon interactions within argon ma-

\footnotetext{
a SUBATECH, UMR CNRS 6457, IN2P3/EMN Nantes/Université de Nantes, 4 Rue A. Kastler, BP 20722, 44307 Nantes Cedex 3, France. E-mail: remi.maurice@subatech.in2p3.fr

${ }^{b}$ CEISAM, UMR CNRS 6230, Université de Nantes, 44322 Nantes Cedex 3, France. E-mail: nicolas.galland@univ-nantes.fr

c PhLAM, UMR CNRS 8523, Université de Lille 1, 59655 Villeneuve d'Ascq Cedex, France $\dagger$ Electronic Supplementary Information (ESI) available: Energies of the $\mathrm{S}_{0}\left({ }^{1} \Delta\right)$ and $\mathrm{S}_{1}\left({ }^{1} \Delta\right)$ electronic states of the $\mathrm{AtO}^{+}\left(\mathrm{H}_{2} \mathrm{O}\right)$ system computed at the NEVPT2/AVTZ level of theory and compositions of the five lowest spin-orbit coupled states at the equilibrium spin-restricted-singlet $\mathrm{AtO}^{+}\left(\mathrm{H}_{2} \mathrm{O}\right)_{n}(n=3-5)$ geometries. See DOI: 10.1039/b000000x/
}

trices. ${ }^{1-4}$ In these cases two electronic states are reversed, which correlate with ${ }^{1} \Sigma^{+}$and ${ }^{3} \Phi$ for CUO, and with ${ }^{3} \Phi_{u}$ and ${ }^{3} \mathrm{H}_{g}$ for $\mathrm{UO}_{2}$. We note that in these two cases, the electronic states involved in the reversal are separated by less than $\sim 0.2 \mathrm{eV}$ in vacuum.

Recently, the $\mathrm{AtO}^{+}$cation was suggested to undergo a solvation-induced ground-state change in aqueous solution. ${ }^{5,6}$ In the gas phase, this cation has a ${ }^{3} \Sigma^{-}$ground spin-orbit-free (SOF) state, separated by $\sim 0.6 \mathrm{eV}$ from the first singlet-spin state, ${ }^{1} \Delta .^{7,8}$ Spin-orbit coupling (SOC) leads to an $X 0^{+}$ground-state that essentially consists of the $M_{S}=0$ component of ${ }^{3} \Sigma^{-}(\sim 70 \%)$ and of ${ }^{1} \Sigma^{+}(\sim 25 \%)$, another SOF singlet-spin state that appears $\sim 1 \mathrm{eV}$ above ${ }^{3} \Sigma^{-}$in the SOF spectrum. ${ }^{7,8}$ However, in aqueous solution, the $\mathrm{AtO}^{+}$cation readily reacts with simple closed-shell anionic species such as $\mathrm{Cl}^{-}, \mathrm{Br}^{-}, \mathrm{SCN}^{-}$and $\mathrm{OH}^{-},{ }^{9-11}$ which implies that the involved reactions are not spin forbidden. Although SOC already leads in the gas phase to a noticeable singlet-spin character in the ground-state of $\mathrm{AtO}^{+}$, a feature which was later found compatible with four-component calculations, ${ }^{7}$ Ayed et al. extensively discussed the role of hydration on the nature of the ground-state of this species by means of relativistic density functional theory (DFT). ${ }^{5,6}$ They hypothesized that hydration induces a ground-state change in $\mathrm{AtO}^{+}$, leading to an essentially-spin- 
singlet SOC ground state. Their work can be the subject of two main critics, (i) the use of single-determinental calculations may lead to incorrect natures of the obtained electronic states, and (ii) the choice of the exchange-correlation functional may not be the most appropriate one. ${ }^{7}$ In this work, we thus aim at clarifying the origin of the non-spin-forbidden complexation that occurs in solution by assessing the respective roles of water molecules belonging to solvation shells and of SOC.

Actually, the $\mathrm{AtO}^{+}$cation is an important species in the astatine Pourbaix $(E-\mathrm{pH})$ diagram, ${ }^{10-13}$ since it can predominate, ${ }^{12}$ and since it is the basic unit of the hydrolyzed $\mathrm{AtO}(\mathrm{OH})$ and AtO $(\mathrm{OH})_{2}^{-}$species that also appear in this diagram. ${ }^{10,11}$ Note that the exploration of the physico-chemical properties of astatine compounds hardly advanced in the last decades due to the experimental difficulties that trigger the radioactive nature and the actual half-life times of its most stable isotopes. ${ }^{14}$ Indeed, the ${ }^{210} \mathrm{At}\left(t_{1 / 2}=8.1 \mathrm{~h}\right)$ and ${ }^{211}$ At $\left(t_{1 / 2}=7.2 \mathrm{~h}\right)$ radionuclides can only be studied at ultra-trace scales, typically after artificial production by irradiation of bismuth targets with alpha beams. ${ }^{15}$ However, since the ${ }^{211} \mathrm{At}$ isotope is recognized as a promising candidate for nuclear medicine, ${ }^{16}$ a strong and recent interest appeared for more fundamental studies on the chemistry of astatine and its compounds. ${ }^{14,17-19}$ In this context, it appears crucial and timely to (i) provide new arguments to justify the occurrence of a hydration-induced ground-state change in $\mathrm{AtO}^{+}$, and (ii) properly characterize the nature of the involved many-electron states, which are the two main objectives of the present work. To avoid the potential issues of the previous studies, ${ }^{5-7}$ we study the stepwise hydration of $\mathrm{AtO}^{+}$with relativistic and multiconfigurational wave-function-based approaches, which proved to be accurate enough to study the zero-field splittings (ZFSs) of various $6 p$ diatomics, ${ }^{8,20}$ as well as to properly discuss the role of SOC on the chemical bonding in these systems. ${ }^{8}$

The paper is organized as follows: first, the computational details are given; then, the results are discussed in three steps, in which we deal with (i) the gas phase electronic structure of $\mathrm{AtO}^{+}$, (ii) the role of solvation on its SOF many-electron states, and (iii) its consequence on the SOC states; finally, conclusions are given.

\section{Computational details}

\subsection{Spin-orbit-free calculations}

The most stable $\mathrm{AtO}^{+}\left(\mathrm{H}_{2} \mathrm{O}\right)_{n} \quad(n=1-6)$ clusters were previously optimized with scalar-relativistic DFT (SR-DFT) and spin-orbit DFT by Ayed et al.. ${ }^{5,6}$ Two types of spin configurations were considered in the SR-DFT calculations, namely spin-restricted-singlet and spin-unrestricted-triplet ones. Note that these earlier studies were done using the M06-2X functional, ${ }^{21}$ a choice that has been recently criticized in the literature. ${ }^{7,22}$ In the present work, we consider the same type of clusters, which have however been optimized with the PBEO hybrid generalized gradient approximation (GGA) functional. ${ }^{23,24}$ We chose this functional since it was previouly proven to provide very accurate geometries for various At-containing systems. ${ }^{22,25}$

The GAUSSIAN program package ${ }^{26}$ was employed to perform all the SR-DFT calculations. The scalar-relativistic many- electron-fit effective core potential ECP60MDF ${ }^{27}$ was used to mimic the role of the 60 core electrons of the At atom. The remaining valence electrons of the At atom were dealt with the aug-cc-pVDZ-PP basis set modified for two-component calculations, ${ }^{9,27,28}$ while for the $\mathrm{H}$ and $\mathrm{O}$ atoms, the aug-cc-pVDZ basis sets were used. ${ }^{29,30}$ For the sake of simplicity, these basis sets will be referred to as "AVDZ" in the remainder of the text. Harmonic vibrational frequencies were determined in order to establish the nature of the obtained structures.

The obtained geometries were used in subsequent wavefunction-based calculations. The state-averaged complete active space self-consistent field (SA-CASSCF) method ${ }^{31,32}$ has been used to build multiconfigurational SOF wave functions. As in previous studies of the $\mathrm{AtO}^{+}$free cation, ${ }^{7,8}$ we have used an active space comprising 8 electrons and 6 molecular orbitals (generated by the $6 p$ and the $2 p$ atomic orbitals of the At and $\mathrm{O}$ centers of $\mathrm{AtO}^{+}$), leading to $\operatorname{CASSCF}(8 / 6)$ calculations. We tested that enlarging the active space by introducing some ligandcentred orbitals does not significantly improve the description of the SOF electronic states of interest. An equal-weighted orbital average between several states of different spin multiplicities has been done ( 6 spin-singlet states, 9 spin-triplet states and 2 spinquintet states). ${ }^{8}$ Note that, in view of subsequent SOC calculations, the state-averaging space cannot be restricted to just a few SOF states. In practice, this state-averaging space must be limited as much as possible while maintaining a good representation of the SOC operator. To recover more electron correlation, we have employed the $n$-electrons valence state perturbation theory at second order (NEVPT2) method in the partially contracted formulation. ${ }^{33}$ Note that the Dyall's Hamiltonian ${ }^{34}$ is used as the zeroth-order Hamiltonian in NEVPT2, which limits the occurrence of intruder states (in comparison with other implementations of multiference second-order perturbation theories).

For all the wave-function-based calculations, we have retained the above-mentioned ECP60MDF effective core potential to mimic the effect of the 60 core electrons of the At atom. Its remaining electrons were dealt with the aug-cc-pVTZ-PP basis set ${ }^{27}$, while for the $\mathrm{H}$ and $\mathrm{O}$ centers we have used the the augcc-pVTZ ones. ${ }^{29,30}$ For the sake of simplicity, these basis sets will be referred to as "AVTZ". In the NEVPT2 calculations, we have frozen the $5 s 5 p 5 d$ semicore shells of the At atom and the $1 s$ core shell of the O one. All the CASSCF and NEVPT2 calculations were performed with the MOLPRO program package. ${ }^{35}$

\subsection{Spin-orbit coupling calculations}

The (relativistic) two-component DFT (2c-DFT) method ${ }^{36}$ implemented in the TURBOMOLE program package ${ }^{37}$ was employed to relax the geometries under the influence of SOC. The previously optimized $\mathrm{AtO}^{+}\left(\mathrm{H}_{2} \mathrm{O}\right)_{n}(n=1-6)$ structures were used as the starting points for the $2 c$-DFT geometry optimizations. For these calculations, we have used the a priori calculated scalarrelativistic wave functions as initial guesses. Naturally, we have retained the aforementioned PBE0 hybrid GGA functional and the AVDZ basis sets. To avoid the treatment of the 60 core electrons of At, we have used the ECP60MDF spin-dependent potential, ${ }^{27}$ 
such that scalar relativistic effects and SOC are both included in the calculations. Numerical frequencies were computed to determine the nature of the obtained $2 c$-DFT structures.

The obtained 2c-DFT geometries were used in subsequent two-step wave-function-based calculations within a contracted spin-orbit configuration interaction (c-SOCI) scheme. SACASSCF (8/6) wave functions were built as zeroth-order wave functions for the SOC calculations, i.e. they are used to compute the off-diagonal matrix elements of the $E_{e l}+\hat{H}_{S O C}$ interaction matrix. The diagonal of this matrix is "dressed" with calculated NEVPT2 energies. The c-SOCI wave functions are obtained by diagonalizing this interaction matrix, which is expressed in terms of the $M_{S}$ components of the considered SOF SA-CASSCF(8/6) solutions. All the c-SOCI calculations were performed with MOLPRO, and the aforementioned ECP60MDF and AVTZ basis sets were used. Note that the SOC integrals have been determined with the spin-dependent part of the ECP60MDF pseudopotential.

\section{Results and discussions}

\subsection{Comments on the $\mathrm{AtO}^{+}$free-cation electronic spectra}

The $\mathrm{AtO}^{+}$free cation exhibits a ${ }^{3} \Sigma^{-}$SOF ground-state that is separated by $\sim 0.6 \mathrm{eV}$ from ${ }^{1} \Delta$ and $\sim 1 \mathrm{eV}$ from ${ }^{1} \Sigma^{+}$. Since in this work we use a different computational setup than the ones used in previous studies, ${ }^{7,8}$ we start by briefly reviewing this case by means of single-point wave-function-based calculations. At the NEVPT2/AVTZ//2c-PBE0/AVDZ level of theory, the ${ }^{3} \Sigma^{-} \rightarrow{ }^{1} \Delta$ and ${ }^{3} \Sigma^{-} \rightarrow{ }^{1} \Sigma^{+}$SOF excitation energies are 0.56 and $1.00 \mathrm{eV}$, respectively. When SOC is introduced, the $\mathrm{X}^{+} \rightarrow \mathbf{a} 1$ and $\mathrm{X}^{+} \rightarrow \mathbf{a}$ 2 excitation energies are 0.42 and $0.98 \mathrm{eV}$, respectively. Since all these values are in good agreement with reference values that are available in the literature, ${ }^{7,8}$ we conclude that no major basis set or geometrical artifact affects the present values, which validates the NEVPT2/AVTZ//2c-PBE0/AVDZ and c-SOCI/AVTZ// $2 c$ $\mathrm{PBE0} / \mathrm{AVDZ}$ levels of theory to study the $\mathrm{AtO}^{+}$free cation.

The following compositions of the SOF wave functions, ${ }^{*}$

$$
\begin{cases}\left|{ }^{3} \Sigma^{-}\right\rangle=\quad & 88 \%\left|[\ldots] \sigma^{2} \pi^{2} \pi^{2} \pi^{* 1} \pi^{* 1} \sigma^{* 0}\right\rangle+\ldots \\ \left|{ }^{1} \Delta\right\rangle= & 42 \%\left|[\ldots] \sigma^{2} \pi^{2} \pi^{2} \pi^{* 1} \pi^{* 1} \sigma^{* 0}\right\rangle \\ & +42 \%\left|[\ldots] \sigma^{2} \pi^{2} \pi^{2} \pi^{* 1} \pi^{* 1} \sigma^{* 0}\right\rangle+\ldots \\ \left|{ }^{1} \Delta\right\rangle{ }^{(2)}= & 42 \%\left|[\ldots] \sigma^{2} \pi^{2} \pi^{2} \pi^{* 2} \pi^{* 0} \sigma^{* 0}\right\rangle \\ & +42 \%\left|[\ldots] \sigma^{2} \pi^{2} \pi^{2} \pi^{* 0} \pi^{* 2} \sigma^{* 0}\right\rangle+\ldots \\ \left|{ }^{1} \Sigma^{+}\right\rangle=\quad & 40 \%\left|[\ldots] \sigma^{2} \pi^{2} \pi^{2} \pi^{* 2} \pi^{* 0} \sigma^{* 0}\right\rangle \\ & +40 \%\left|[\ldots] \sigma^{2} \pi^{2} \pi^{2} \pi^{* 0} \pi^{* 2} \sigma^{* 0}\right\rangle+\ldots\end{cases}
$$

and of the SOC wave functions,

\footnotetext{
* In the determinental expansion of the wave function corresponding to the $\left|{ }^{1} \Delta\right\rangle^{(2)}$
} state, the second CSF that is shown carries a coefficient with opposite sign.

$$
\left\{\begin{array}{l}
\left.\left|X 0^{+}\right\rangle=\left.68 \%\right|^{3} \Sigma^{-}\right\rangle_{M_{S}=0}+26 \%\left|{ }^{1} \Sigma^{+}\right\rangle+\ldots \\
\left.\left.|\mathbf{a} 1\rangle^{(1,2)}=\left.43 \%\right|^{3} \Sigma^{-}\right\rangle_{M_{S}=+1}+\left.43 \%\right|^{3} \Sigma^{-}\right\rangle_{M_{S}=-1}+\ldots \\
\left.|\mathbf{a} 2\rangle^{(1)}=\left.83 \%\right|^{1} \Delta\right\rangle^{(1)}+\ldots \\
\left.|\mathbf{a} 2\rangle^{(2)}=\left.83 \%\right|^{1} \Delta\right\rangle^{(2)}+\ldots
\end{array}\right.
$$

have been obtained for the lowest-lying electronic states of interest. Again, we note a good agreement of the NEVPT2/AVTZ//2cPBE0/AVDZ and c-SOCI/AVTZ//2c-PBE0/AVDZ wave functions with the reference ones that are available in the literature. ${ }^{7,8}$ If $\mathrm{AtO}^{+}$undergoes a ground-state change in water, leading to a closed-shell singlet-spin ground-state as suggested by Ayed et al. , 5,6 two main hypotheses can be formulated from eqs. 1 and 2, (i) a closed-shell configuration, correlating most likely with one component of ${ }^{1} \Delta$, becomes the SOF ground-state and most contribute to the ground SOC state, or (ii) a closed-shell configuration is stabilized in such a way that it does not become the SOF ground-state while being the most important contribution to the ground SOC state. We will first discriminate between these two hypotheses by monitoring the electronic states that correlate with ${ }^{3} \Sigma^{-},{ }^{1} \Delta$ and ${ }^{1} \Sigma^{+}$in different $\mathrm{AtO}^{+}\left(\mathrm{H}_{2} \mathrm{O}\right)_{n}(n=1-6)$ clusters by means of NEVPT2/AVTZ calculations. After this, using c-SOCI/AVTZ calculations, we will scrutinize the SOC states to firmly justify the occurrence of a hydration-induced ground-state change in $\mathrm{AtO}^{+}$.

\subsection{Spin-orbit-free calculations}

In this section we discuss the electronic structures of the $\mathrm{AtO}^{+}\left(\mathrm{H}_{2} \mathrm{O}\right)_{n} \quad(n=1-6)$ systems by means of single-point NEVPT2/AVTZ calculations performed at the PBE0/AVDZ geometries. For each system, two geometries are considered, corresponding to the equilibrium structures of the lowest-energy spinrestricted-singlet and spin-unrestricted-triplet SR-DFT solutions (see Fig. 1). Note that more extensive discussions concerning these geometries can be found elsewhere. ${ }^{5,6}$ We just recall briefly that the hydration spheres corresponding to the different spinsymmetry solutions for each system significantly differ. For instance, in the spin-restricted-singlet clusters, the water molecules lead to charge transfer to At and may form hydrogen bonds with the $\mathrm{O}$ atom of the $\mathrm{AtO}^{+}$unit. In contrast, the conformation of the surrounding water molecules in the spin-unrestricted-triplet clusters is ruled by halogen bonding with At, while hydrogen bonds are only formed between water units. Moreover, the interatomic distances are different in both types of clusters, with shorter water-AtO ${ }^{+}$distances in the spin-restricted-singlet clusters. We will split the discussion in two parts, by first analyzing the $\mathrm{AtO}^{+}\left(\mathrm{H}_{2} \mathrm{O}\right)$ system, and then discussing the $\mathrm{AtO}^{+}\left(\mathrm{H}_{2} \mathrm{O}\right)_{n}$ $(n=2-6)$ cases. Note that SOF excitation energies corresponding to all the studied clusters are reported in Table 1.

\subsubsection{The $\mathrm{AtO}^{+}\left(\mathrm{H}_{2} \mathrm{O}\right)$ system}

In agreement with the previous M06-2X/AVDZ studies, ${ }^{5,6}$ we find that the equilibrium geometry for the spin-unrestricted-triplet cluster belongs to the $\mathrm{C}_{2 \mathrm{v}}$ symmetry point group, while the spinrestricted-singlet one has a $\mathrm{C}_{\mathrm{s}}$ symmetry. At first, we check that 


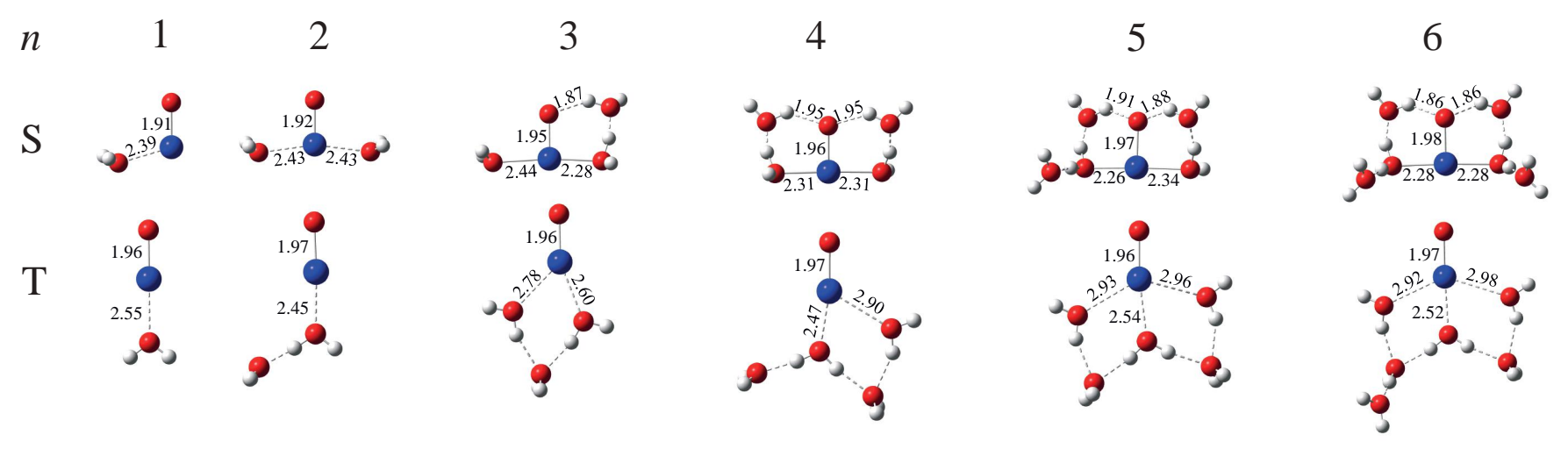

Fig. 1 PBE0/AVDZ lowest-energy structures for the spin-restricted-singlet (top) and spin-unrestricted-triplet (bottom) AtO ${ }^{+}\left(\mathrm{H}_{2} \mathrm{O}\right)_{n}$ clusters $(n=1-6)$. Interatomic distances are given in Angstroms. Color code: blue stands for At and red for $\mathrm{O}$.

Table 1 Calculated scalar-relativistic excitation energies (in eV) for the different equilibrium structures displayed in Fig. 1 . The $\Delta E$ (non-adiabatic) energy difference between the spin-restricted-singlet state and the spin-restricted-triplet state is also given in each case (a positive value means that the spin-unrestricted-triplet cluster is more stable).

\begin{tabular}{|c|c|c|c|c|c|c|c|}
\hline & & $\mathrm{AtO}^{+}\left(\mathrm{H}_{2} \mathrm{O}\right)$ & $\mathrm{AtO}^{+}\left(\mathrm{H}_{2} \mathrm{O}\right)_{2}$ & $\mathrm{AtO}^{+}\left(\mathrm{H}_{2} \mathrm{O}\right)_{3}$ & $\mathrm{AtO}^{+}\left(\mathrm{H}_{2} \mathrm{O}\right)_{4}$ & $\mathrm{AtO}^{+}\left(\mathrm{H}_{2} \mathrm{O}\right)_{5}$ & $\mathrm{AtO}^{+}\left(\mathrm{H}_{2} \mathrm{O}\right)_{6}$ \\
\hline \multicolumn{8}{|c|}{ Spin-unrestricted-triplet clusters } \\
\hline \multirow[t]{4}{*}{ NEVPT2/AVTZ } & $\mathrm{T}_{0}\left({ }^{3} \Sigma^{-}\right)$ & 0.00 & 0.00 & 0.00 & 0.00 & 0.00 & 0.00 \\
\hline & $\mathrm{S}_{0}\left({ }^{1} \Delta\right)$ & 0.54 & 0.53 & 0.45 & 0.48 & 0.30 & 0.29 \\
\hline & $\mathrm{S}_{1}\left({ }^{1} \Delta\right)$ & 0.55 & 0.54 & 0.54 & 0.54 & 0.54 & 0.54 \\
\hline & $\mathrm{S}_{2}\left({ }^{1} \Sigma^{+}\right)$ & 0.97 & 0.96 & 1.09 & 1.02 & 1.24 & 1.25 \\
\hline \multicolumn{8}{|c|}{ Spin-restricted-singlet clusters } \\
\hline \multirow[t]{4}{*}{ NEVPT2/AVTZ } & $\mathrm{T}_{0}\left({ }^{3} \Sigma^{-}\right)$ & 0.17 & 1.09 & 1.76 & 2.26 & 2.45 & 2.71 \\
\hline & $\mathrm{S}_{0}\left({ }^{1} \Delta\right)$ & 0.00 & 0.00 & 0.00 & 0.00 & 0.00 & 0.00 \\
\hline & $\mathrm{S}_{1}\left({ }^{1} \Delta\right)$ & 0.70 & 1.58 & 2.25 & 2.80 & 3.00 & 3.40 \\
\hline & $\mathrm{S}_{2}\left({ }^{1} \Sigma^{+}\right)$ & 2.08 & 4.01 & $>4.97^{a}$ & $>5.42^{a}$ & $>5.60^{a}$ & $>5.76^{a}$ \\
\hline \multicolumn{8}{|c|}{ Energy differences } \\
\hline NEVPT2/AVTZ & $\Delta E$ & $0.55(0.57)^{b}$ & $0.23(0.26)^{b}$ & $-0.02(-0.03)^{b}$ & -0.26 & -0.52 & -0.64 \\
\hline
\end{tabular}

${ }^{a}$ When $\mathrm{S}_{2}\left({ }^{1} \Sigma^{+}\right)$is out of the state-averaging space, we only report a lower limit for the corresponding excitation energy.

${ }^{b}$ The values in parenthesis are calculated at the NEVPT2/AVTZ//PBE0/AVTZ level of theory. These values show that there is no bias introduced by the use of equilibrium geometries calculated with the smaller AVDZ basis set.

these SR-DFT stationary points nearly correspond to stationary points of the NEVPT2/AVTZ potential energy surfaces. For this, we study the dependence of the energy of various states with respect to the two main coordinates that are at play, i.e. the $\mathrm{H}_{2} \mathrm{O}-\mathrm{AtO}^{+}$bond distance and the $\angle \mathrm{OAtO}$ bond angle. We fix all the other geometrical parameters to the values they have in the PBE0/AVDZ spin-unrestricted-triplet structure. As can be seen in Figs. 2 and $S 1$ (where only $S_{0}$ and $S_{1}$ are displayed for a sake of clarity), we found a spin-restricted-triplet $\mathrm{C}_{2 \mathrm{v}}$ minimum, a spinrestricted-singlet $C_{2 v}$ minimum, and a spin-restricted-singlet $C_{s}$ minimum, the latter being the global minimum for the singlet multiplicity.

Fig. 2A evidences that the lowest-energy electronic states correlate with the states of the free $\mathrm{AtO}^{+}$cation: one spin-restrictedtriplet state correlate with ${ }^{3} \Sigma^{-}$, two spin-restricted-singlet roots correlate with ${ }^{1} \Delta$, and one spin-restricted-singlet state correlates with ${ }^{1} \Sigma^{+}$. Therefore, we label these states as $\mathrm{T}_{0}\left({ }^{3} \Sigma^{-}\right)$, $S_{0}\left({ }^{1} \Delta\right), S_{1}\left({ }^{1} \Delta\right)$ and $S_{2}\left({ }^{1} \Sigma^{+}\right)$. Note that at the PBE0/AVDZ spinunrestricted-triplet geometry, the degeneracy lift between $\mathrm{S}_{0}\left({ }^{1} \Delta\right)$ and $S_{1}\left({ }^{1} \Delta\right)$ is very small $(\sim 0.01 \mathrm{eV})$, as expected. It can be seen from Fig. 2B that the $S_{2}\left({ }^{1} \Sigma^{+}\right)$state is largely destabilized while going toward the equilibrium $\mathrm{C}_{s}$ geometry. Also, due to the de- generacy lifting of the frontier $\pi^{*}$ orbitals, the $S_{1}\left({ }^{1} \Delta\right)-S_{0}\left({ }^{1} \Delta\right)$ energy gap increases when one considers a minimum energy path between the $\mathrm{C}_{2 \mathrm{v}}$ and $\mathrm{C}_{\mathrm{s}}$ geometries. Indeed, while going to the spin-restricted-singlet geometry, the $\pi^{*}$ orbital that belongs to the OAtO plane is relatively destabilized with respect to the other one because of the ligand-field created by the water molecule.

At the equilibrium PBE0/AVDZ $\mathrm{C}_{\mathrm{s}}$ geometry, the $\mathrm{T}_{0}\left({ }^{3} \Sigma^{-}\right)$, $\mathrm{S}_{1}\left({ }^{1} \Delta\right)$ and $S_{2}\left({ }^{1} \Sigma^{+}\right)$states lie $0.17,0.70$ and $2.08 \mathrm{eV}$ above $\mathrm{S}_{0}\left({ }^{1} \Delta\right)$, respectively (see Table 1 ). The CASSCF wave function of the $S_{0}\left({ }^{1} \Delta\right)$ state is essentially constituted of the $[\ldots] \sigma^{2} \pi^{2} \pi^{2} \pi^{* 2} \pi^{* 0} \sigma^{* 0}$ configuration (81\%), the $\mathrm{T}_{0}\left({ }^{3} \Sigma^{-}\right)$state of the $[\ldots] \sigma^{2} \pi^{2} \pi^{2} \pi^{* 1} \pi^{* 1} \sigma^{* 0}$ configuration (87\%), the $S_{1}\left({ }^{1} \Delta\right)$ state of the $[\ldots] \sigma^{2} \pi^{2} \pi^{2} \pi^{* 1} \pi^{* 1} \sigma^{* 0}$ configuration (81\%), and the $S_{2}\left({ }^{1} \Sigma^{+}\right)$ state of the [...] $\sigma^{2} \pi^{2} \pi^{2} \pi^{* 0} \pi^{* 2} \sigma^{* 0}$ configuration (82\%). Since at this geometry the $S_{0}\left({ }^{1} \Delta\right)$ state is the ground-state, the premise of the hydration-induced ground-state change are already observed in the $\mathrm{AtO}^{+}\left(\mathrm{H}_{2} \mathrm{O}\right)$ system. However, the spin-unrestrictedtriplet cluster is more stable than the spin-restricted-singlet one by $0.55 \mathrm{eV}$ (see Table 1). Therefore, one has to pursue the micro-hydration in order to determine if $\mathrm{AtO}^{+}$undergoes an hydration-induced ground-state change at the scalar-relativistic NEVPT2/AVTZ level. 
A
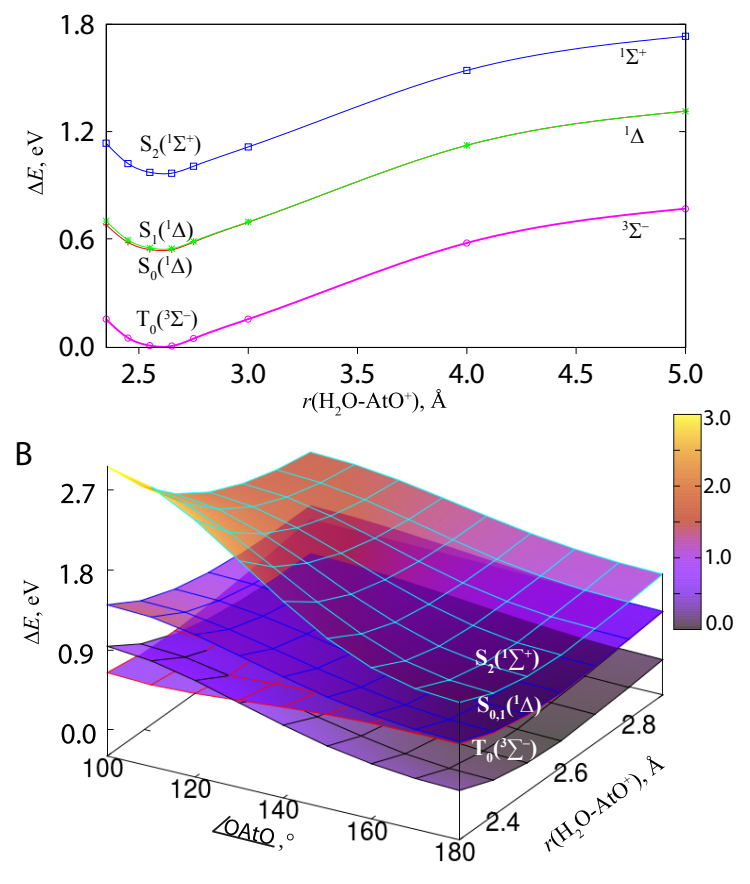

Fig. 2 Evolution of the relative energies of the lowest-lying electronic states of the $\mathrm{AtO}^{+}\left(\mathrm{H}_{2} \mathrm{O}\right)$ system computed at the NEVPT2/AVTZ level of theory as a function of the $\mathrm{H}_{2} \mathrm{O}-\mathrm{AtO}^{+}$distance and of the $/ \mathrm{OAtO}$ angle; A: the angle is fixed to $180^{\circ}$ while the distance is varied; $\mathrm{B}$ : both the angle and the distance are varied.

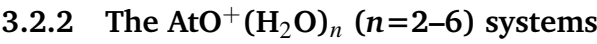

We start by discussing the nature of the electronic states that correlate with the ${ }^{3} \Sigma^{-},{ }^{1} \Delta$ and ${ }^{1} \Sigma^{+}$of the free $\mathrm{AtO}^{+}$cation. At the spin-unrestricted-triplet geometries, these states are not strongly perturbed by hydration since the water units tend to create a network between themselves through hydrogen bonding (see Fig. 1). For all these structures, we find that $\mathrm{T}_{0}\left({ }^{3} \Sigma^{-}\right)$is the groundstate. The corresponding CASSCF wave functions in each case reveal that they are mainly single-configurational, with weights of more than $80 \%$ on the $[\ldots] \sigma^{2} \pi^{2} \pi^{2} \pi^{* 1} \pi^{* 1} \sigma^{* 0}$ configuration. In each case, we have identified three spin-restricted-singlet states right above $\mathrm{T}_{0}\left({ }^{3} \Sigma^{-}\right)$. The first two of them are nearly degenerate in the $n=2$ case, and the energy gap between them increases with increasing $n$, up to $0.25 \mathrm{eV}$ in the $n=6$ case (see Table 1 ). The CASSCF wave functions of the lowest-energy ones have, in all the cases, $\sim 80 \%$ weights on the closed-shell $[. ..] \sigma^{2} \pi^{2} \pi^{2} \pi^{* 2} \pi^{* 0} \sigma^{* 0}$ and [...] $\sigma^{2} \pi^{2} \pi^{2} \pi^{* 0} \pi^{* 2} \sigma^{* 0}$ configurations. When $n$ is increased, due to the degeneracy lift of the $\pi^{*}$ orbitals, the weight of the former configuration increases up to $78 \%$ for these states, which thus becomes essentially single-configurational. The CASSCF wave functions of the second spin-restricted-singlet roots have $\sim 80 \%$ weight on the $[\ldots] \sigma^{2} \pi^{2} \pi^{2} \pi^{* 1} \pi^{* 1} \sigma^{* 0}$ configuration. Thus, these sets of states clearly correlate with the ${ }^{1} \Delta$ state of the free $\mathrm{AtO}^{+}$ cation. The CASSCF wave functions corresponding to the third spin-restricted-singlet root have $\sim 80 \%$ weights on the closed- shell [...] $\sigma^{2} \pi^{2} \pi^{2} \pi^{* 2} \pi^{* 0} \sigma^{* 0}$ and [...] $\sigma^{2} \pi^{2} \pi^{2} \pi^{* 0} \pi^{* 2} \sigma^{* 0}$ configurations, with the weight of the latter configuration increasing with $n$. Therefore, these third spin-restricted-singlet roots correlate with ${ }^{1} \Sigma^{+}$, and we note that these states are relatively destabilized with increasing $n$ (see Table 1).

We now continue by analyzing the electronic states of the spinrestricted-singlet equilibrium structures. As in the $\mathrm{AtO}^{+}\left(\mathrm{H}_{2} \mathrm{O}\right)$ spin-restricted-singlet case, the obtained spectra are expected to largely differ from the one of the $\mathrm{AtO}^{+}$free cation. Obviously, one cannot play with all the degrees of freedom that arise from the water positions to track states from the free $\mathrm{AtO}^{+}$cation to each $\mathrm{AtO}^{+}\left(\mathrm{H}_{2} \mathrm{O}\right)_{n}$ structure of interest. A simple approach consists in calculating the lowest-lying electronic states at the equilibrium geometries corresponding to these spin-restricted-singlet clusters and to back-trace them up to dissociating the water molecules. We monitored the evolution of the states correlating with ${ }^{3} \Sigma^{-},{ }^{1} \Delta$, and ${ }^{1} \Sigma^{+}$while the 2(6) water molecules are moving away in the $\mathrm{C}_{2 \mathrm{v}}\left(\mathrm{C}_{\mathrm{s}}\right)$ symmetry point group (see Fig. 3). Note that in the $n=6$ case, the parameters which are scanned correspond to the two bonds between the water molecules and the At center (see the spin-singlet $\mathrm{AtO}^{+}\left(\mathrm{H}_{2} \mathrm{O}\right)_{6}$ cluster in Fig. 1), the 6 water molecules moving simultaneously away from the $\mathrm{AtO}^{+}$unit in a symmetric fashion. Notably, the closed-shell electronic state that correlate with ${ }^{1} \Delta$, which we label as $S_{0}\left({ }^{1} \Delta\right)$, crosses $T_{0}\left({ }^{3} \Sigma^{-}\right)$at distances bellow $\sim 3.0 \AA$ and becomes the ground-state for shorter distances such that it reaches its minimum at distances smaller than $\sim 2.5$ $\AA$ A. Note that the differential stabilization of $\mathrm{S}_{0}\left({ }^{1} \Delta\right)$ and $\mathrm{T}_{0}\left({ }^{3} \Sigma^{-}\right)$ with increasing $n$ can be easily explained: the energy gap between the two orbitals that correlate with $\pi^{*}$ increases with increasing $n$; therefore, it costs more energy to promote an electron to the other $\pi^{*}$ orbital than to pair two electrons in the lowest-energy $\pi^{*}$ orbital to form the closed-shell configuration.

In all the $\mathrm{AtO}^{+}\left(\mathrm{H}_{2} \mathrm{O}\right)_{n}(n=2-6)$ spin-restricted-singlet clusters, the CASSCF ground-state wave functions are dominated by the closed-shell [...] $\sigma^{2} \pi^{2} \pi^{2} \pi^{* 2} \pi^{* 0} \sigma^{* 0}$ configuration (with weights larger than $80 \%)$. The states labeled as $\mathrm{T}_{0}\left({ }^{3} \Sigma^{-}\right)$are always the first excited states and the corresponding excitation energies increase with increasing $n$ (see Table 1). Their CASSCF wave functions are essentially single-configurational and have weights larger than $80 \%$ on the $[\ldots] \sigma^{2} \pi^{2} \pi^{2} \pi^{* 1} \pi^{* 1} \sigma^{* 0}$ configuration. The $\mathrm{S}_{1}\left({ }^{1} \Delta\right)$ states are also relatively destabilized with increasing $n$ (see Table 1), as their CASSCF wave functions have weights larger than $82 \%$ on the $[\ldots] \sigma^{2} \pi^{2} \pi^{2} \pi^{* 1} \pi^{* 1} \sigma^{* 0}$ configuration. We found that the $S_{2}\left({ }^{1} \Sigma^{+}\right)$states are so much destabilized with increasing $n$ that starting from $n=3$, they go out of the used state-averaging space, which is not surprising since this root essentially corresponds to the $[\ldots] \sigma^{2} \pi^{2} \pi^{2} \pi^{* 0} \pi^{* 2} \sigma^{* 0}$ configuration.

From Table 1, it is clear that the spin-restricted-singlet clusters quickly become more stable than the spin-unrestricted-triplet ones when $n$ is increased. Therefore, at the SOF level, hydration induces a ground-state reversal between $\mathrm{T}_{0}\left({ }^{3} \Sigma^{-}\right)$and $\mathrm{S}_{0}\left({ }^{1} \Delta\right)$, and therefore, one of the two aforementioned hypotheses can be dropped out. We thus conclude from this part that hydration induces a ground-state change at the SOF level, which supports the results of Ayed et. al. ${ }^{5,6}$ 

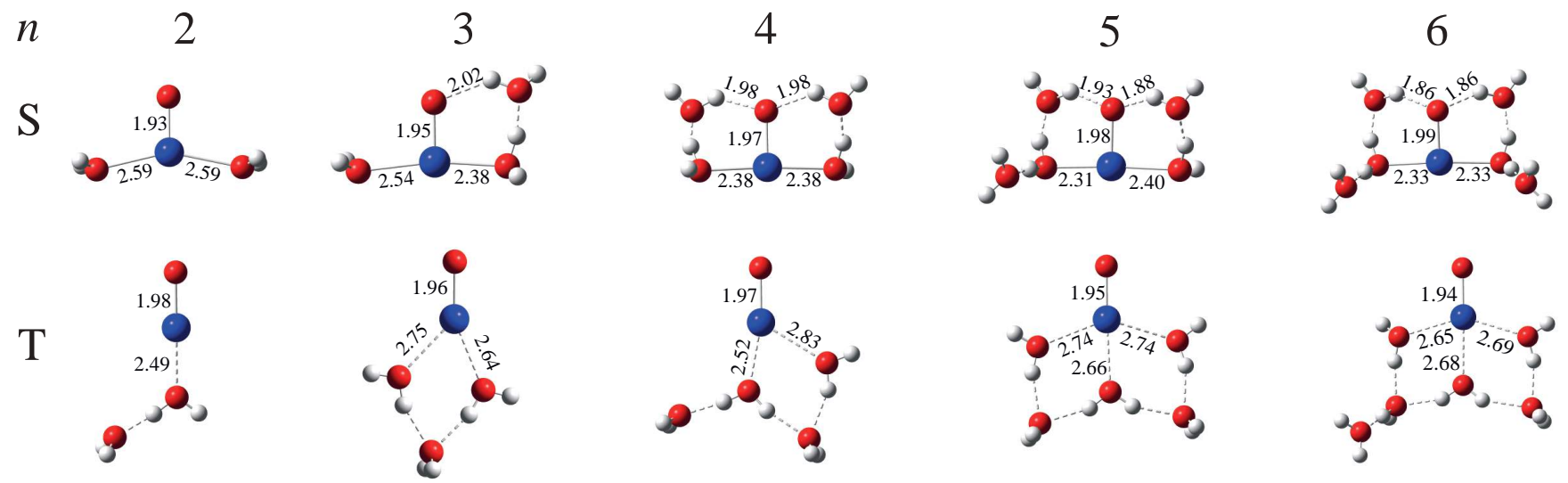

Fig. 4 2c-PBE0/AVDZ lowest-energy structures for the essentially-spin-singlet (top) and essentially-spin-triplet (bottom) AtO ${ }^{+}\left(\mathrm{H}_{2} \mathrm{O}\right)_{n}$ clusters $(n=2-6)$. Interatomic distances are given in Angstroms. Color code: blue stands for At and red for $\mathrm{O}$.

\subsection{Spin-orbit coupling calculations}

In this section we discuss the influence of SOC on the geometries and electronic structures of the essentially-spin-triplet and essentially-spin-singlet $\mathrm{AtO}^{+}\left(\mathrm{H}_{2} \mathrm{O}\right)_{n}(n=1-6)$ clusters. The geometries optimized for the $\mathrm{AtO}^{+}\left(\mathrm{H}_{2} \mathrm{O}\right)_{n}(n=2-6)$ systems the $2 c-$ PBE0/AVDZ level of theory are shown in Fig. 4. The vertical excitation energies obtained at the NEVPT2/AVTZ//2c-PBE0/AVDZ and c-SOCI/AVTZ//2c-PBE0/AVDZ levels are gathered in Table 2. Note that the $n=1$ case will be discussed separately as it deserves special attention. The influence of SOC on the geometries of the two types of clusters is different: while in the essentiallyspin-singlet clusters the water-AtO ${ }^{+}$distances generally increase, in some of the essentially-spin-triplet clusters such water-AtO ${ }^{+}$ distances can decrease (see Figs. 1 and 4). Therefore, SOC is susceptible for strengthening specific water- $\mathrm{AtO}^{+}$interactions in these clusters.

\subsubsection{The $\mathrm{AtO}^{+}\left(\mathrm{H}_{2} \mathrm{O}\right)$ system}

In line with previous results, ${ }^{6,7}$ we found at the $2 c$-PBE0/AVDZ level of theory that the equilibrium geometry of the essentiallyspin-triplet $\mathrm{AtO}^{+}\left(\mathrm{H}_{2} \mathrm{O}\right)$ cluster belongs to the $\mathrm{C}_{2 \mathrm{v}}$ symmetry point group, with a larger $\mathrm{H}_{2} \mathrm{O}-\mathrm{AtO}^{+}$distance than the one obtained at the SOF level (i.e. $2.63 \AA$ A). We have not identified a $\mathrm{C}_{\text {s-like equi- }}$ librium geometry for the essentially-spin-singlet cluster. Therefore, we performed c-SOCI calculations in order to study the dependence of the energy and the nature of the lowest-lying SOC states, with respect to the $\mathrm{H}_{2} \mathrm{O}-\mathrm{AtO}^{+}$distance and to the $\angle \mathrm{OAtO}$ angle (see Fig. 5). In order to identify these states, we have tracked them while the water molecule approaches toward the essentially-spin-triplet equilibrium geometry (in a similar way as what was done at the scalar-relativistic level). As expected, they clearly correlate with the $X 0^{+}$, a 1 and a 2 states of the free $\mathrm{AtO}^{+}$cation, and therefore, we label them accordingly. As can be seen in Fig. 5A, the five lowest-lying SOC states all raise in energy toward the region of the potential energy surface where the equilibrium scalar-relativistic spin-singlet geometry was found.

At first, we focus on the $\Psi\left(X 0^{+}\right)$SOC ground-state and the evolution of its composition as a function of $\mathrm{H}_{2} \mathrm{O}-\mathrm{AtO}^{+}$distance and the $\angle \mathrm{OAtO}$ angle (see Fig. $5 \mathrm{~B}$ ). At the essentially-spin-triplet equilibrium geometry $\left(\mathrm{C}_{2 \mathrm{v}}\right)$, this state is very similar to the one of the free $\mathrm{AtO}^{+}$cation: it is essentially composed of the $M_{S}=0$ component of $\mathrm{T}_{0}\left({ }^{3} \Sigma^{-}\right)(68 \%)$ and of $\mathrm{S}_{2}\left({ }^{1} \Sigma^{+}\right)(25 \%)$. Since the $\mathrm{T}_{0}\left({ }^{3} \Sigma^{-}\right)$and $\mathrm{S}_{2}\left({ }^{1} \Sigma^{+}\right)$states are relatively destabilized when going toward the scalar-relativistic spin-singlet equilibrium geometry (see Fig. 2B), their contributions to the $\Psi\left(X^{+} 0^{+}\right)$groundstate wave function is diminished (see Fig. 5B). In contrast, as $\mathrm{S}_{0}\left({ }^{1} \Delta\right)$ is at the same time relatively stabilized (see Fig. $2 \mathrm{~B}$ ), its contribution to $\Psi\left(\mathrm{X}^{+}\right)$is enhanced (see Fig. 5B). At the scalarrelativistic spin-singlet equilibrium geometry $\left(\mathrm{C}_{\mathrm{S}}\right)$, we find that the ground SOC state $\left(\Psi_{0}\right)$ has a mixed singlet-triplet character, with $45 \%$ of $\mathrm{T}_{0}\left({ }^{3} \Sigma^{-}\right), 5 \%$ of $\mathrm{S}_{2}\left({ }^{1} \Sigma^{+}\right)$and $41 \%$ of $\mathrm{S}_{0}\left({ }^{1} \Delta\right)$.

The two $\Psi($ a 1$)$ states lie $\sim 0.40 \mathrm{eV}$ above $\Psi\left(X^{+} 0^{+}\right)$at the essentially-spin-triplet geometry $\left(\mathrm{C}_{2 \mathrm{v}}\right)$ and are mainly composed of $M_{S}=-1(43 \%)$ and $M_{S}=+1(43 \%)$ components of $\mathrm{T}_{0}\left({ }^{3} \Sigma^{-}\right)$. Toward the scalar-relativistic spin-singlet geometry $\left(\mathrm{C}_{\mathrm{s}}\right)$, these states raise in energy and appear at $0.67\left(\Psi_{1}\right)$ and $0.68 \mathrm{eV}\left(\Psi_{2}\right)$ above the ground SOC state while keeping their compositions. Since these states are not involved in the ground-state change under study, we continue by discussing the evolution of the $\Psi$ (a 2) states. At the essentially-spin-triplet geometry, they lie $\sim 0.95 \mathrm{eV}$ above $\Psi\left(X^{+} 0^{+}\right)$. Their wave functions are essentially composed of the $S_{0}\left({ }^{1} \Delta\right)$ and $S_{1}\left({ }^{1} \Delta\right)$ states ( $82 \%$ and $83 \%$, respectively). Toward the scalar-relativistic spin-singlet geometry, the energy gap between the two $\Psi(\mathbf{a} 2)$ states gradually increases (see Fig. 5A), these states being at $0.93\left(\Psi_{3}\right)$ and $1.20 \mathrm{eV}\left(\Psi_{4}\right)$ above $\Psi_{0}$ at this geometry. The composition of the $\Psi_{3}$ SOC state shows a decrease in its $\mathrm{S}_{0}\left({ }^{1} \Delta\right)$ component from $82 \%$ in the essentially-spin-triplet geometry $\left(\mathrm{C}_{2 \mathrm{v}}\right)$ to $46 \%$ in the scalar-relativistic spin-singlet structure, while the weight of the $M_{S}=0$ component of the $\mathrm{T}_{0}\left({ }^{3} \Sigma^{-}\right)$ state increases from 0 to $31 \%$. At the same time, the composition of the $\Psi_{4}$ state is almost unaffected. Note that the $\mathrm{C}_{\mathrm{s}}$ geometry does not correspond to a minimum for neither $\Psi_{3}$ nor $\Psi_{4}$ (see figure $5 \mathrm{~A}$ ), which explains that we failed in optimizing an essentially-spin-singlet structure at the 2c-PBE0 level. As stated by Ayed et al., 5,6 a fierce competition between SOC and hydration occurs, the former being dominant in the $\mathrm{AtO}^{+}\left(\mathrm{H}_{2} \mathrm{O}\right)$ system. As a consequence, no essentially-spin-singlet structure can 
Table 2 Calculated electronic excitation energies (in eV) for the different equilibrium structures displayed in Fig. 4. The $\Delta E$ (non-adiabatic) energy difference between the essentially-spin-singlet state and the essentially-spin-triplet state is also given in each case (a positive value means that the essentially-spin-triplet state is more stable).

\begin{tabular}{|c|c|c|c|c|c|c|}
\hline & & $\mathrm{AtO}^{+}\left(\mathrm{H}_{2} \mathrm{O}\right)_{2}$ & $\mathrm{AtO}^{+}\left(\mathrm{H}_{2} \mathrm{O}\right)_{3}$ & $\mathrm{AtO}^{+}\left(\mathrm{H}_{2} \mathrm{O}\right)_{4}$ & $\mathrm{AtO}^{+}\left(\mathrm{H}_{2} \mathrm{O}\right)_{5}$ & $\mathrm{AtO}^{+}\left(\mathrm{H}_{2} \mathrm{O}\right)_{6}$ \\
\hline \multicolumn{7}{|c|}{ Essentially-spin-triplet clusters } \\
\hline \multirow[t]{4}{*}{ NEVPT2/AVTZ } & $\mathrm{T}_{0}\left({ }^{3} \Sigma^{-}\right)$ & 0.00 & 0.00 & 0.00 & 0.01 & 0.21 \\
\hline & $\mathrm{S}_{0}\left({ }^{1} \Delta\right)$ & 0.53 & 0.43 & 0.44 & 0.00 & 0.00 \\
\hline & $S_{1}\left({ }^{1} \Delta\right)$ & 0.54 & 0.54 & 0.54 & 0.54 & 0.74 \\
\hline & $\mathrm{S}_{2}\left({ }^{1} \Sigma^{+}\right)$ & 0.95 & 1.10 & 1.06 & 1.63 & 2.08 \\
\hline \multirow[t]{5}{*}{ c-SOCI/NEVPT2/AVTZ } & $\Psi_{0}$ & 0.00 & 0.00 & 0.00 & 0.00 & 0.00 \\
\hline & $\Psi_{1}$ & 0.37 & 0.43 & 0.44 & 0.62 & 0.71 \\
\hline & $\Psi_{2}$ & 0.37 & 0.44 & 0.45 & 0.64 & 0.74 \\
\hline & $\Psi_{3}$ & 0.91 & 0.97 & 0.96 & 1.01 & 1.03 \\
\hline & $\Psi_{4}$ & 0.91 & 0.98 & 0.97 & 1.14 & 1.22 \\
\hline \multicolumn{7}{|c|}{ Essentially-spin-singlet clusters } \\
\hline \multirow[t]{4}{*}{ NEVPT2/AVTZ } & $\mathrm{T}_{0}\left({ }^{3} \Sigma^{-}\right)$ & 0.50 & 1.14 & 1.80 & 2.08 & 2.28 \\
\hline & $\mathrm{S}_{0}\left({ }^{1} \Delta\right)$ & 0.00 & 0.00 & 0.00 & 0.00 & 0.00 \\
\hline & $\mathrm{S}_{1}\left({ }^{1} \Delta\right)$ & 1.01 & 1.63 & 2.33 & 2.61 & 2.81 \\
\hline & $\mathrm{S}_{2}\left({ }^{1} \Sigma^{+}\right)$ & 2.74 & 3.94 & $>4.90^{a}$ & $>5.16^{a}$ & $>5.34^{a}$ \\
\hline \multirow[t]{5}{*}{ c-SOCI/NEVPT2/AVTZ } & $\Psi_{0}$ & 0.00 & 0.00 & 0.00 & 0.00 & 0.00 \\
\hline & $\Psi_{1}$ & 0.89 & 1.44 & 2.04 & 2.21 & 2.31 \\
\hline & $\Psi_{2}$ & 0.89 & 1.44 & 2.12 & 2.33 & 2.45 \\
\hline & $\Psi_{3}$ & 1.05 & 1.47 & 2.21 & 2.36 & 2.48 \\
\hline & $\Psi_{4}$ & 1.37 & 1.83 & 2.34 & 2.45 & 2.51 \\
\hline \multicolumn{7}{|l|}{ Energy differences } \\
\hline c-SOCI/NEVPT2/AVTZ & $\Delta E$ & $0.33(0.32)^{b}$ & $0.30(0.30)^{b}$ & 0.10 & -0.10 & -0.23 \\
\hline
\end{tabular}

${ }^{a}$ When $\mathrm{S}_{2}\left({ }^{1} \Sigma^{+}\right)$is out of the state-averaging space, we only report a lower limit for the corresponding excitation energy.

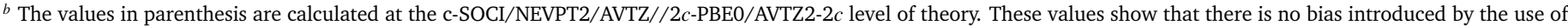
equilibrium geometries calculated with the smaller AVDZ basis set.

be obtained in this system.

\subsubsection{The $\mathrm{AtO}^{+}\left(\mathrm{H}_{2} \mathrm{O}\right)_{n}(n=2-6)$ systems}

At first, we discuss the energy levels obtained at the essentiallyspin-triplet geometries of the $\mathrm{AtO}^{+}\left(\mathrm{H}_{2} \mathrm{O}\right)_{n} \quad(n=2-6)$ clusters. While we noticed only little changes in the SOF states for $n \leq 4$, a ground-state reversal between $\mathrm{T}_{0}\left({ }^{3} \Sigma^{-}\right)$and $\mathrm{S}_{0}\left({ }^{1} \Delta\right)$ occurs in the $n=5-6$ cases. One should note that this ground-state reversal did not occur at the scalar-relativistic spin-triplet geometries, meaning that the geometrical changes induced by SOC matter. In Table 2 we have denoted as $\Psi_{n}, n=0-4$, the lowest five SOC states. At the equilibrium geometry of the $n=2$ essentially-spin-triplet cluster, the compositions of the c-SOCI wave functions of these states read:

$$
\left\{\begin{array}{l}
\left|\Psi_{0}\right\rangle=69 \%\left|\mathrm{~T}_{0}\left({ }^{3} \Sigma^{-}\right)\right\rangle_{M_{S}=0}+24 \%\left|\mathrm{~S}_{2}\left({ }^{1} \Sigma^{+}\right)\right\rangle+\ldots \\
\left|\Psi_{1}\right\rangle=43 \%\left|\mathrm{~T}_{0}\left({ }^{3} \Sigma^{-}\right)\right\rangle_{M_{S}=+1}+43 \%\left|\mathrm{~T}_{0}\left({ }^{3} \Sigma^{-}\right)\right\rangle_{M_{S}=-1}+\ldots \\
\left|\Psi_{2}\right\rangle=43 \%\left|\mathrm{~T}_{0}\left({ }^{3} \Sigma^{-}\right)\right\rangle_{M_{S}=+1}+43 \%\left|\mathrm{~T}_{0}\left({ }^{3} \Sigma^{-}\right)\right\rangle_{M_{S}=-1}+\ldots \\
\left|\Psi_{3}\right\rangle=81 \%\left|\mathrm{~S}_{0}\left({ }^{1} \Delta\right)\right\rangle+\ldots \\
\left|\Psi_{4}\right\rangle=82 \%\left|\mathrm{~S}_{1}\left({ }^{1} \Delta\right)\right\rangle+\ldots
\end{array}\right.
$$

In terms of composition, they clearly correlate with the lowest five SOC states of the free $\mathrm{AtO}^{+}$cation. $\Psi_{0}$ has a dominant spin-triplet character and correlates with the $\mathrm{XO}^{+}$state of $\mathrm{AtO}^{+}$. The nearlydegenerate $\Psi_{1}$ and $\Psi_{2}$ states are placed $0.37 \mathrm{eV}$ above $\Psi_{0}$; they have dominant spin-triplet characters and correlate with the twofold degenerate a 1 state of $\mathrm{AtO}^{+}$. The nearly-degenerate $\Psi_{3}$ and $\Psi_{4}$ states, $0.91 \mathrm{eV}$ above $\Psi_{0}$, have dominant spin-singlet charac- ter and correlate with the two-fold degenerate a 2 state of $\mathrm{AtO}^{+}$. We note that the vertical excitation energies to these $\Psi_{n}(n=1-$ 4) states are very similar to the ones observed in the free $\mathrm{AtO}^{+}$ cation, suggesting that the two water molecules do not perturb that much the $\mathrm{AtO}^{+}$unit. While $n$ increases, the near degeneracies between the $\Psi_{1}$ and $\Psi_{2}$ and between the $\Psi_{3}$ and $\Psi_{4}$ states are more and more lifted, all these SOC states being destabilized with respect to the $\Psi_{0}$ states (see Table 2). Also, the composition of the corresponding SOC wave functions evolve with $n$. For the $n=6$ essentially-spin-triplet cluster, the compositions of the c-SOCI wave functions are:

$$
\left\{\begin{aligned}
\left|\Psi_{0}\right\rangle= & 46 \%\left|\mathrm{~T}_{0}\left({ }^{3} \Sigma^{-}\right)\right\rangle_{M_{S}=0}+40 \%\left|\mathrm{~S}_{0}\left({ }^{1} \Delta\right)\right\rangle+\ldots \\
\left|\Psi_{1}\right\rangle= & 43 \%\left|\mathrm{~T}_{0}\left({ }^{3} \Sigma^{-}\right)\right\rangle_{M_{S}=+1}+43 \%\left|\mathrm{~T}_{0}\left({ }^{3} \Sigma^{-}\right)\right\rangle_{M_{S}=-1}+\ldots \\
\left|\Psi_{2}\right\rangle= & 44 \%\left|\mathrm{~T}_{0}\left({ }^{3} \Sigma^{-}\right)\right\rangle_{M_{S}=+1}+44 \%\left|\mathrm{~T}_{0}\left({ }^{3} \Sigma^{-}\right)\right\rangle_{M_{S}=-1}+\ldots \\
\left|\Psi_{3}\right\rangle= & 29 \%\left|\mathrm{~T}_{0}\left({ }^{3} \Sigma^{-}\right)\right\rangle_{M_{S}=0}+14 \%\left|\mathrm{~S}_{2}\left({ }^{1} \Sigma^{+}\right)\right\rangle \\
& +46 \%\left|\mathrm{~S}_{0}\left({ }^{1} \Delta\right)\right\rangle+\ldots \\
\left|\Psi_{4}\right\rangle= & 84 \%\left|\mathrm{~S}_{1}\left({ }^{1} \Delta\right)\right\rangle+\ldots
\end{aligned}\right.
$$

By comparing the c-SOCI wave functions shown in Eq. 3 and Eq. 4, it is clear that the $\Psi_{1}, \Psi_{2}$ and the $\Psi_{4}$ states are nearly unchanged while $n$ is increased from 2 to 6 . On the other hand, interesting changes appear in the composition of the $\Psi_{0}$ and $\Psi_{3}$ states: with increasing $n$, the $\left(M_{S}=0\right) \mathrm{T}_{0}\left({ }^{3} \Sigma^{-}\right)$contribution to the $\Psi_{0}$ wave function is decreased while the one of the $\Psi_{3}$ one is increased, and the $S_{0}\left({ }^{1} \Delta\right)$ contribution to $\Psi_{3}$ is decreased while the one of $\Psi_{0}$ is increased. In the $n=6$ essentially-spin-triplet 


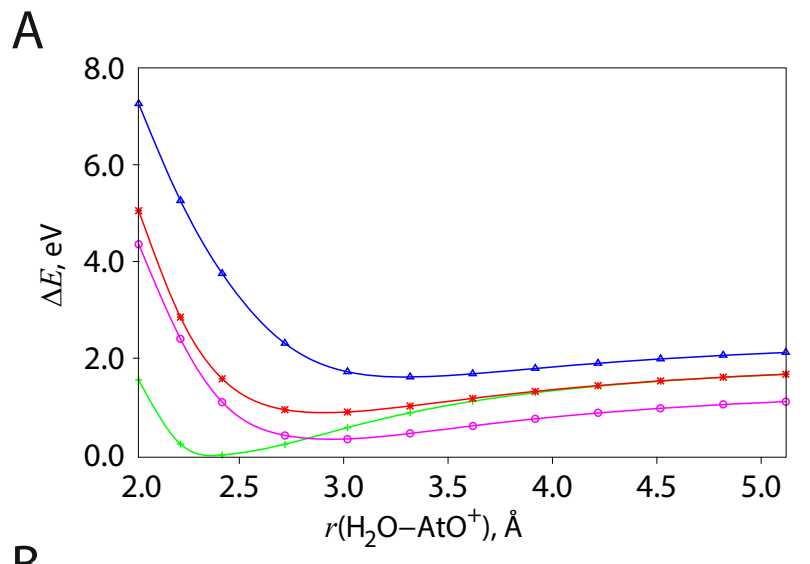

B

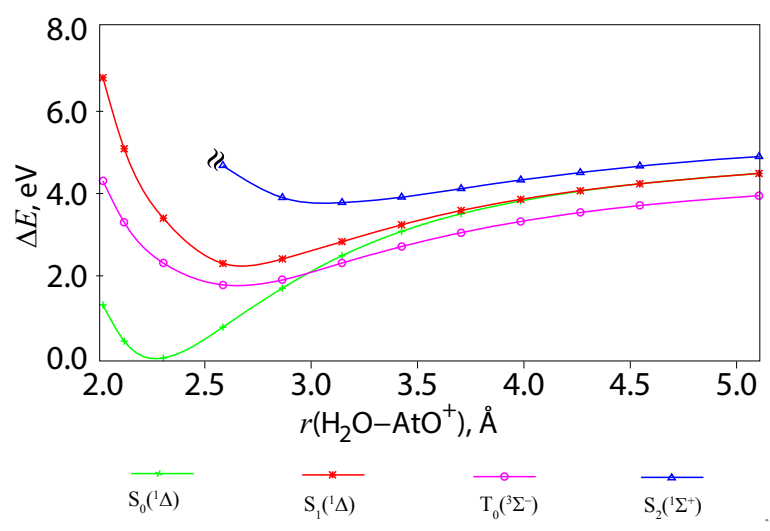

Fig. 3 Evolution of the energy of low-lying electronic states as a function of the main $\mathrm{H}_{2} \mathrm{O}-\mathrm{AtO}^{+}$distances; $\mathrm{A}$ : in the $\mathrm{AtO}^{+}\left(\mathrm{H}_{2} \mathrm{O}\right)_{2}$ system $\left(\mathrm{C}_{2 v}\right.$ symmetry); $\mathrm{B}$ : in the $\mathrm{AtO}^{+}\left(\mathrm{H}_{2} \mathrm{O}\right)_{6}$ system $\left(\mathrm{C}_{\mathrm{s}}\right.$ symmetry). The geometries of the spin-restricted-singlet clusters are taken as references.

cluster, the $\Psi_{0}$ and $\Psi_{3}$ states have mixed singlet/triplet characters (see eq. 4). The concerted change in the dominant compositions of the $\Psi_{0}$ and $\Psi_{3}$ wave functions is in line with the change in the relative energies of the corresponding SOF states (see Table 2).

Now, we discuss the energy levels obtained at the essentiallyspin-singlet geometries. Following the geometrical changes induced SOC, we noticed that the SOF states are largely affected in terms of relative energies: the $S_{0}\left({ }^{1} \Delta\right)$ states remain the groundstates in each case while the vertical excitation energies from these states to the $\mathrm{T}_{0}\left({ }^{3} \Sigma^{-}\right)$and $\mathrm{S}_{1}\left({ }^{1} \Delta\right)$ ones are significantly reduced (see Tables 1 and 2). The compositions of the c-SOCI wave functions for the $n=2$ case read:

$$
\left\{\begin{array}{l}
\left|\Psi_{0}\right\rangle=34 \%\left|\mathrm{~T}_{0}\left({ }^{3} \Sigma^{-}\right)\right\rangle_{M_{S}=0}+53 \%\left|\mathrm{~S}_{0}\left({ }^{1} \Delta\right)\right\rangle+\ldots \\
\left|\Psi_{1}\right\rangle=43 \%\left|\mathrm{~T}_{0}\left({ }^{3} \Sigma^{-}\right)\right\rangle_{M_{S}=+1}+43 \%\left|\mathrm{~T}_{0}\left({ }^{3} \Sigma^{-}\right)\right\rangle_{M_{S}=-1}+\ldots \\
\left|\Psi_{2}\right\rangle=43 \%\left|\mathrm{~T}_{0}\left({ }^{3} \Sigma^{-}\right)\right\rangle_{M_{S}=+1}+43 \%\left|\mathrm{~T}_{0}\left({ }^{3} \Sigma^{-}\right)\right\rangle_{M_{S}=-1}+\ldots \\
\left|\Psi_{3}\right\rangle=42 \%\left|\mathrm{~T}_{0}\left({ }^{3} \Sigma^{-}\right)\right\rangle_{M_{S}=0}+35 \%\left|\mathrm{~S}_{0}\left({ }^{1} \Delta\right)\right\rangle+\ldots \\
\left|\Psi_{4}\right\rangle=83 \%\left|\mathrm{~S}_{1}\left({ }^{1} \Delta\right)\right\rangle+\ldots
\end{array}\right.
$$

The $\Psi_{1}$ and $\Psi_{2}$ states are degenerate in energy and they lie at $0.89 \mathrm{eV}$ above the ground-state. In terms of composition, these

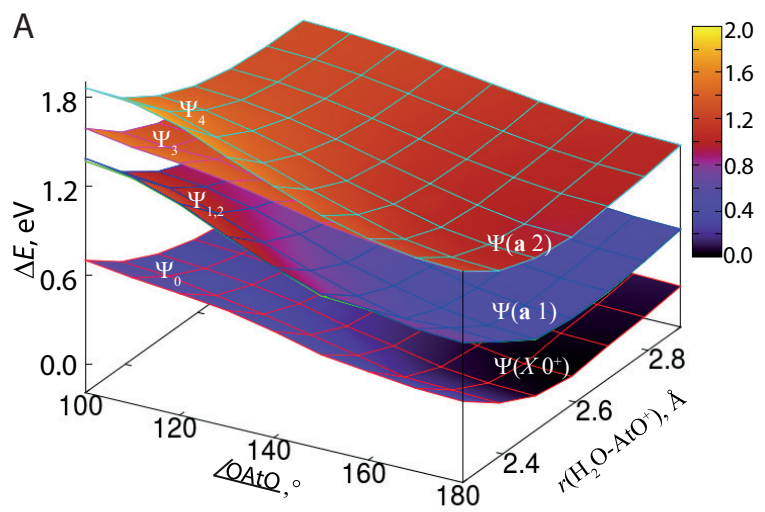

B

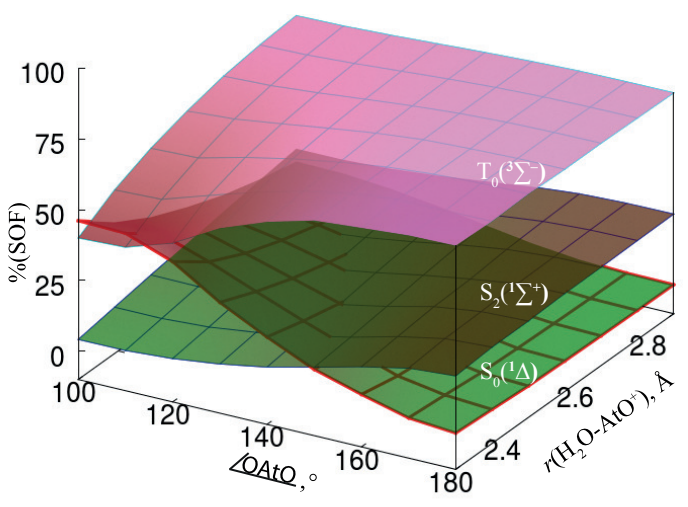

Fig. 5 Evolution of the lowest-lying energy levels of the $\mathrm{AtO}^{+}\left(\mathrm{H}_{2} \mathrm{O}\right)$ system computed at the CSOCI/AVTZ level of theory as a function of the $\mathrm{H}_{2} \mathrm{O}-\mathrm{AtO}^{+}$distance and of the $\angle \mathrm{OAtO}$ angle; $\mathrm{A}$ : relative energies (in $\mathrm{eV})$; B: composition of $\Psi_{0}$ in terms of the $M_{S}$ components of $\mathrm{T}_{0}\left({ }^{3} \Sigma^{-}\right)$, $\mathrm{S}_{0}\left({ }^{1} \Delta\right)$, and $\mathrm{S}_{2}\left({ }^{1} \Sigma^{+}\right)$.

states have dominant spin-triplet characters and clearly correlate with the two-fold degenerate a 1 state of $\mathrm{AtO}^{+} . \Psi_{4}$ lies $1.37 \mathrm{eV}$ above the ground-state, its wave function has a dominant spinsinglet character (it correlates with the open-shell component of a 2). The wave functions of $\Psi_{0}$ and $\Psi_{3}$ have mixed singlet/triplet characters instead of dominant spin-triplet and spin-singlet characters, respectively, as was the case at the $n=2$ essentially-spintriplet equilibrium geometry (see Eq. 2). The compositions of the c-SOCI wave functions for the $n=6$ case are:

$$
\left\{\begin{aligned}
\left|\Psi_{0}\right\rangle= & 84 \%\left|\mathrm{~S}_{0}\left({ }^{1} \Delta\right)\right\rangle+\ldots \\
\left|\Psi_{1}\right\rangle= & 28 \%\left|\mathrm{~T}_{0}\left({ }^{3} \Sigma^{-}\right)\right\rangle_{M_{S}=+1}+28 \%\left|\mathrm{~T}_{0}\left({ }^{3} \Sigma^{-}\right)\right\rangle_{M_{S}=-1} \\
& +42 \%\left|\mathrm{~T}_{1}\left({ }^{3} \Pi\right)\right\rangle_{M_{S}=0}+\ldots \\
\left|\Psi_{2}\right\rangle= & 21 \%\left|\mathrm{~T}_{1}\left({ }^{3} \Pi\right)\right\rangle_{M_{S}=+1}+21 \%\left|\mathrm{~T}_{1}\left({ }^{3} \Pi\right)\right\rangle_{M_{S}=-1} \\
& +42 \%\left|\mathrm{~T}_{0}\left({ }^{3} \Sigma^{-}\right)\right\rangle_{M_{S}=0}+\ldots \\
\left|\Psi_{3}\right\rangle= & 39 \%\left|\mathrm{~T}_{0}\left({ }^{3} \Sigma^{-}\right)\right\rangle_{M_{S}=+1}+39 \%\left|\mathrm{~T}_{0}\left({ }^{3} \Sigma^{-}\right)\right\rangle_{M_{S}=-1}+\ldots \\
\left|\Psi_{4}\right\rangle= & 35 \%\left|\mathrm{~S}_{1}\left({ }^{1} \Delta\right)\right\rangle+31 \%\left|\mathrm{~T}_{1}\left({ }^{3} \Pi\right)\right\rangle_{M_{S}=+1} \\
& +31 \%\left|\mathrm{~T}_{1}\left({ }^{3} \Pi\right)\right\rangle_{M_{S}=-1}+\ldots
\end{aligned}\right.
$$

As a consequence of the stabilization of the $S_{0}\left({ }^{1} \Delta\right)$ SOF state with respect to the $\mathrm{T}_{0}\left({ }^{3} \Sigma^{-}\right)$one, $\Psi_{0}$ as a dominant spin-singlet character, i.e. $84 \%$ of its wave function corresponds to the $\mathrm{S}_{0}\left({ }^{1} \Delta\right)$ state. This $\Psi_{0}$ state clearly correlate with the closed-shell com- 
ponent of the a 2 state of the free $\mathrm{AtO}^{+}$cation. The remaining SOC states, namely $\Psi_{1}, \Psi_{2}, \Psi_{3}$ and $\Psi_{4}$, are more and more destabilized with respect to $\Psi_{0}$ with increasing $n$ (see Table 2). Actually, when going from the $n=2$ to the $n=6$ essentially-spin-singlet cluster, the composition of their wave functions gradually change (see Eq. S1). For instance, in the $n=6$ case, the wave functions corresponding to $\Psi_{1}, \Psi_{2}$ and $\Psi_{3}$ exhibit mixtures of the $M_{S}$ components of the lowest two SOF triplet states, $\mathrm{T}_{0}\left({ }^{3} \Sigma^{-}\right)$and $\mathrm{T}_{1}\left({ }^{3} \Pi\right)$, while the one of $\Psi_{4}$ exhibits a mixture of $S_{1}\left({ }^{1} \Delta\right)$ and the $\left|M_{S}\right|=1$ components of $\mathrm{T}_{1}\left({ }^{3} \Pi\right)$.

Finally, we discuss the nature of the ground-states of the $\mathrm{AtO}^{+}\left(\mathrm{H}_{2} \mathrm{O}\right)_{n} \quad(n=2-6)$ systems. Clearly, beyond $n=4$ water molecules in the first hydration sphere, the lowest-energy structures exhibit essentially-spin-singlet closed-shell ground-states. Therefore, when SOC is included in the calculations, $\mathrm{AtO}^{+}$undergoes a hydration-induced ground-state change, leading to an essentially closed-shell ground-state that can readily react in a spin-allowed fashion with closed-shell species. Note that in this work, we have not taken into consideration a continuum solvation model to introduce long-range bulk effects. Nonetheless, these effects have been lengthy discussed by Ayed et. $a l^{5,6}$ and were shown to strongly stabilize the $\mathrm{AtO}^{+}$-water species that exhibit (essentially-)spin-singlet ground-states relatively to the ones exhibiting (essentially-)spin-triplet ones. Our results are thus in qualitative agreement with this trend. Therefore, we confirm the recent M06-2X results of Ayed et. al ${ }^{5,6}$ and complements them by clearly establishing the nature of the electronic states that are involved in this ground-state change.

\section{Conclusions}

Although recently suggested, the fact that $\mathrm{AtO}^{+}$undergoes a hydration-induced ground-state change was recently questioned in the literature. ${ }^{7}$ In this work, we have used relativistic density functional theory and wave function theory methods to revisit this phenomenon. While in the gas-phase and in the absence of spin-orbit coupling, AtO ${ }^{+}$adopts a spin-triplet ground-state $\left({ }^{3} \Sigma^{-}\right)$, we showed that hydration leads to a closed-shell groundstate, even when relatively small hydration spheres $(n=3$ and beyond) are considered. We have determined for the first time the nature of these states, showing that they clearly correlate with the closed-shell component of free-cation ${ }^{1} \Delta$ state. When spinorbit coupling is accounted for, $\mathrm{AtO}^{+}$adopts, in the gas-phase, an essentially-spin-triplet ground-state, $X^{+} 0^{+}$, with a strong weight on the $M_{S}=0$ component of the ${ }^{3} \Sigma^{-}$SOF state. As a consequence of the hydration effects, the $\mathrm{AtO}^{+}\left(\mathrm{H}_{2} \mathrm{O}\right)_{n}(n \geq 5)$ clusters adopt essentially-spin-singlet closed-shell ground-states. We have determined, for the first time, that these ground-states are dominated by the closed-shell $\mathrm{S}_{0}\left({ }^{1} \Delta\right)$, originating from the closed-shell component of ${ }^{1} \Delta$ of the free $\mathrm{AtO}^{+}$cation. We showed that this spinorbit-coupled state correlate with one component of the a 2 state of $\mathrm{AtO}^{+}$, meaning that a hydration-induced ground-state change definitely occurs in $\mathrm{AtO}^{+}$, which supports the studies of Ayed et al. ${ }^{5,6}$

The $\mathrm{AtO}^{+}$species is one of the cationic forms that appears to predominate in acidic conditions in the Pourbaix $(E-\mathrm{pH})$ diagram of astatine. This species readily reacts with closed- shell species, which can be explained by the abovementioned hydration-induced ground-state change. The fact that it undergoes a ground-state change in aqueous solution is clearly a peculiarity which further shows once more that the chemistry of astatine and its species can be very different from the one of the other halogens. ${ }^{10,25,38-40}$ Therefore, it remains crucial and timely to pursue chemical studies concerning this "invisible" element.

Acknowledgments This work has been supported by grants funded by the French National Agency for Research with "Investissements d'Avenir" (ANR-11-EQPX-0004, ANR-11-LABX0018). This work was performed using HPC resources from CCIPL ("Centre de Calcul Intensif des Pays de la Loire").

\section{References}

1 L. Andrews, B. Liang, J. Li and B. E. Bursten, Angew. Chem., 2000, 112, 4739-4741.

2 J. Li, B. E. Bursten, B. Liang and L. Andrews, Science, 2002, 295, 2242-2245.

3 J. Li, B. E. Bursten, L. Andrews and C. J. Marsden, J. Am. Chem. Soc., 2004, 126, 3424-3425.

4 I. Infante, L. Andrews, X. Wang and L. Gagliardi, Chem. Eur. $J, 2010,16,12804-12807$.

5 T. Ayed, M. Seydou, F. Réal, G. Montavon and N. Galland, $J$. Phys. Chem. B, 2013, 117, 5206-5211.

6 T. Ayed, F. Réal, G. Montavon and N. Galland, J. Phys. Chem. B, 2013, 117, 10589-10595.

7 A. S. P. Gomes, F. Réal, N. Galland, C. Angeli, R. Cimiraglia and V. Vallet, Chem. Phys. Phys. Chem., 2014, 16, 9238-9248.

8 R. Maurice, F. Réal, A. S. P. Gomes, V. Vallet, G. Montavon and N. Galland, J. Chem. Phys., 2015, 142, 094305.

9 J. Champion, M. Seydou, A. Sabatié-Gogova, E. Renault, G. Montavon and N. Galland, Phys. Chem. Chem. Phys., 2011, 13, 14984-14992.

10 J. Champion, A. Sabatié-Gogova, F. Bassal, T. Ayed, C. Alliot, N. Galland and G. Montavon, J. Phys. Chem. A, 2013, 117, 1983-1990.

11 D.-C. Sergentu, D. Teze, A. Sabatié-Gogova, C. Alliot, N. Guo, F. Bassal, I. Da Silva, D. Deniaud, R. Maurice, J. Champion, N. Galland and G. Montavon, Chem. Eur. J., 2016, 22, 2964 2971.

12 J. Champion, C. Alliot, E. Renault, B. M. Mokili, M. Chérel, N. Galland and G. Montavon, J. Phys. Chem. A, 2010, 114, 576-582.

13 A. Sabatié-Gogova, J. Champion, S. Huclier, N. Michel, F. Pottier, N. Galland, Z. Asfari, M. Cherél and G. Montavon, Anal. Chim. Acta, 2012, 721, 182-188.

14 D. S. Wilbur, Nat. Chem., 2013, 5, 246.

15 M. R. Zalutsky and M. Pruszynski, Curr. Radiopharm., 2011, 4, 177-185.

16 D. S. Wilbur, Curr. Radiopharm., 2008, 1, 144-176.

17 S. G. DiMagno, 2014, US Patent: US20140275539 A1.

18 M. Amaouch, G. Montavon, N. Galland and J. Pilmé, Mol. Phys., 2016, 114, 1326-1333. 
19 T. Ayed, J. Pilmé, D. Tézé, F. Bassal, J. Barbet, M. Chérel, J. Champion, R. Maurice, G. Montavon and N. Galland, Eur. J. Med. Chem., 2016, 116, 156-164.

20 J.-B. Rota, S. Knecht, T. Fleig, D. Ganyushin, T. Saue, F. Neese and H. Bolvin, J. Chem. Phys., 2011, 135, 114106.

21 Y. Zhao and D. G. Truhlar, Theor. Chem. Acc, 2008, 120, 215241.

22 D.-C. Sergentu, G. David, G. Montavon, R. Maurice and N. Galland, J. Comput. Chem., 2016, 37, 1345-1354.

23 J. P. Perdew, M. Ernzerhof and K. Burke, J. Chem. Phys., 1996, 105, 9982-9985.

24 C. Adamo and V. Barone, J. Chem. Phys., 1999, 110, 61586170.

25 D.-C. Sergentu, M. Amouch, J. Pilmé, N. Galland and R. Maurice, J. Chem. Phys., 2015, 143, 114306.

26 M. J. Frisch, G. W. Trucks, H. B. Schlegel, G. E. Scuseria, M. A. Robb, J. R. Cheeseman, G. Scalmani, V. Barone, B. Mennucci, G. A. Petersson, H. Nakatsuji, M. Caricato, X. Li, H. P. Hratchian, A. F. Izmaylov, J. Bloino, G. Zheng, J. L. Sonnenberg, M. Hada, M. Ehara, K. Toyota, R. Fukuda, J. Hasegawa, M. Ishida, T. Nakajima, Y. Honda, O. Kitao, H. Nakai, T. Vreven, J. A. Montgomery, Jr., J. E. Peralta, F. Ogliaro, M. Bearpark, J. J. Heyd, E. Brothers, K. N. Kudin, V. N. Staroverov, R. Kobayashi, J. Normand, K. Raghavachari, A. Rendell, J. C. Burant, S. S. Iyengar, J. Tomasi, M. Cossi, N. Rega, J. M. Millam, M. Klene, J. E. Knox, J. B. Cross, V. Bakken, C. Adamo, J. Jaramillo, R. Gomperts, R. E. Stratmann, O. Yazyev, A. J. Austin, R. Cammi, C. Pomelli, J. W. Ochterski, R. L. Martin, K. Morokuma, V. G. Zakrzewski, G. A. Voth, P. Salvador, J. J. Dannenberg, S. Dapprich, A. D. Daniels, Ã. Farkas, J. B. Foresman, J. V. Ortiz, J. Cioslowski and D. J. Fox, Gaussian 09 Revision D.01, Gaussian Inc. Wallingford CT 2009.

27 K. A. Peterson, D. Figgen, E. Goll, H. Stoll and M. Dolg, J. Chem. Phys., 2003, 119, 11113-11123.

28 M. K. Armbruster, K. W. and F. Weigend, Phys. Chem. Chem. Phys., 2006, 8, 4862-4865.

29 T. H. Dunning Jr, J. Chem. Phys., 1989, 90, 1007-1023.

30 R. A. Kendall, T. H. Dunning and R. J. Harrison, J. Chem. Phys., 1992, 96, 6796-6806.

31 B. O. Roos, P. R. Taylor and P. E. M. Siegbahn, Chem. Phys., 1980, 48, 157-173.

32 B. O. Roos, in Theory and applications of computational chemistry: The first forty years, ed. C. E. Dykstra, G. Frenking, K. S. Kim and G. E. Scuseria, Elsevier, Amsterdam, 2005, ch. 25, pp. 725-764.

33 A. Celestino, R. Cimiraglia and J.-P. Malrieu, Chem. Phys. Lett., 2001, 350, 297-305.

34 K. G. Dyall, J. Chem. Phys., 1995, 102, 4909-4918.

35 H. J. Werner, P. J. Knowles, R. Lindh, F. R. Manby, M. Schütz, P. Celani, T. Korona, A. Mitrushenkov, G. Rauhut, T. B. Adler, R. D. Amos, A. Bernhardsson, A. Berning, D. L. Cooper, M. J. O. Deegan, A. J. Dobbyn, F. Eckert, E. Goll, C. Hampel, G. Hetzer, T. Hrenar, G. Knizia, C. Köppl, Y. Liu, A. W.
Lloyd, R. A. Mata, A. J. May, S. J. McNicholas, W. Meyer, M. E. Mura, A. Nicklass, P. Palmieri, K. Pflüger, R. Pitzer, M. Reiher, U. Schumann, H. Stoll, A. J. Stone, R. Tarroni, T. Thorsteinsson, M. Wang and A. Wolf, MOLPRO, Version 2009.1, A Package of Ab Initio Programs, 2009, see http://www.molpro.net.

36 M. K. Armbruster, F. Weigend, C. van Wullen and W. Klopper, Phys. Chem. Chem. Phys., 2008, 10, 1748-1756.

37 TURBOMOLE V6.6 2014, A Development of University of Karlsruhe And Forschungszentrum Karlsruhe GmbH, 1989-2007, TURBOMOLE GmbH, since 2007; available from http: / / www.turbomole.com.

38 J. Pilmé, E. Renault, T. Ayed, G. Montavon and N. Galland, J. Chem. Theory Comput., 2012, 8, 2985-2990.

39 A. Hermann, R. Hoffmann and N. W. Ashcroft, Phys. Rev. Lett., 2013, 111, 116404-116409.

40 R. E. Vernon, J. Chem. Educ., 2013, 90, 1703-1707. 


\section{Table of contents:}

Artwork:

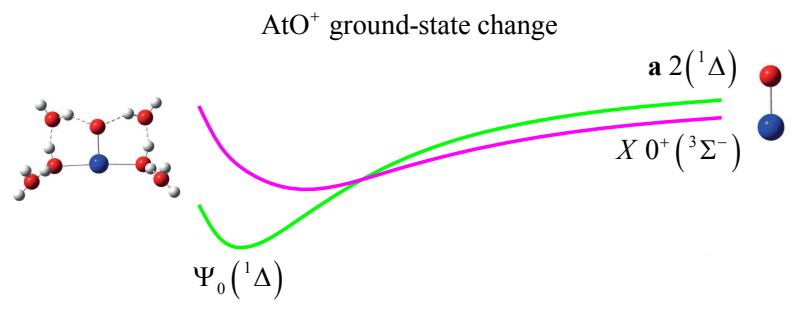

\section{Synopsis:}

The hydration-induced ground-state change of $\mathrm{AtO}^{+}$is confirmed by means of relativistic and multiconfigurational wave-functionbased calculations. The involved spin-orbit-free states are found to correlate with ${ }^{3} \Sigma^{-}$and ${ }^{1} \Delta$, while the spin-orbit-coupled ones correlate with $\mathrm{XO}^{+}$and a 2. Therefore, the complexation of $\mathrm{AtO}^{+}$ with closed-shell ligands is not spin forbidden. 